Portland State University

PDXScholar

Electrical and Computer Engineering Faculty

Publications and Presentations

Electrical and Computer Engineering

2-13-2017

\title{
Ideal Radial Permanent Magnet Coupling Torque Density Analysis
}

\author{
Kang Li \\ University of North Carolina at Charlotte \\ Jonathan Bird \\ Portland State University \\ Vedanadam M. Acharya \\ University of North Carolina at Charlotte
}

Follow this and additional works at: https://pdxscholar.library.pdx.edu/ece_fac

Part of the Electrical and Computer Engineering Commons

Let us know how access to this document benefits you.

\section{Citation Details}

Li, Kang; Bird, Jonathan; and Acharya, Vedanadam M., "Ideal Radial Permanent Magnet Coupling Torque Density Analysis" (2017). Electrical and Computer Engineering Faculty Publications and Presentations.

420.

https://pdxscholar.library.pdx.edu/ece_fac/420

This Post-Print is brought to you for free and open access. It has been accepted for inclusion in Electrical and Computer Engineering Faculty Publications and Presentations by an authorized administrator of PDXScholar. Please contact us if we can make this document more accessible: pdxscholar@pdx.edu. 


\title{
Ideal Radial Permanent Magnet Coupling Torque Density Analysis
}

\author{
Kang Li (李康) $)^{1}$, Student Member, IEEE, Jonathan Z. Bird ${ }^{2}$ Member, IEEE, Vedanadam M. Acharya ${ }^{1}$ \\ ${ }^{1}$ University of North Carolina at Charlotte, Department of Electrical and Computer Engineering, Charlotte, NC, USA \\ ${ }^{2}$ Portland State University, Department of Electrical and Computer Engineering, Portland, OR, USA
}

\begin{abstract}
This paper derives the closed form 3-D analytical torque equations for an ideal radial Halbach rotor magnetic coupling. The performance of the radial Halbach coupling is then compared with an ideal axial Halbach rotor coupling. The closed form equations and comparison gives insight into the upper torque density limits of Nd-Fe-B based magnetic devices.
\end{abstract}

Index Terms - Analytical models, axial coupling, electromagnetic forces, Halbach rotor, radial coupling

\section{INTRODUCTION}

$\mathrm{A}^{\mathrm{p}}$ PERMANENT magnetic coupling (PMC) creates synchronous torque transmission without physical contact. The study of the torque capabilities of magnetic couplings is fundamental to ones understand of the upper bound on the torque densities of magnetic devices, such as motors and magnetic gearboxes [1]. The axial and radial PMC, as illustrated in Fig. 1, form the two primary types of PMCs. The external field created by a PMC can be made close to sinusoidal in form if a Halbach rotor magnet structure is utilized. An example of an axial and radial Halbach rotor magnet arrangement is shown in Fig. 2. In order to compute the PMCs torque the field from each individual magnet is typically first determined and then the magnet's fields are summed up and used to compute the torque. Examples of authors using this approach are given in [2-5]. Frédéric [5] for instance, then used such a technique to study the performance of different magnetic couplings that utilize rectangular magnets. Other authors have utilized the finite element analysis (FEA) technique to determine the optimal geometric design for a PMC $[6,7]$.

In this paper the radial PMC torque is derived using a magnetic charge sheet approach that enables the torque equation to be expressed by a single integral without the need for a large number of summations. The radial PMC torque density characteristics are then compared with the performance capabilities of an equivalently sized axial PMC [8]. The accuracy of the magnetic charge modelling approach is validated by using 3-D FEA. The use of the analytic based modeling and idealized assumptions enabled fundamental geometric scaling parameter relationships to be identified.

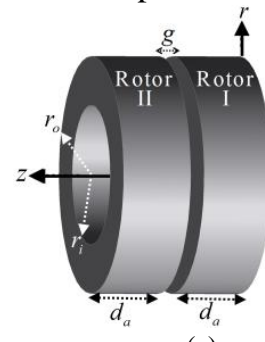

(a)

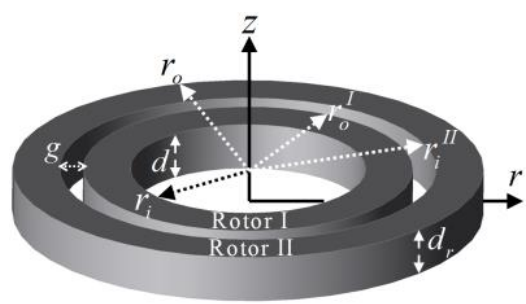

(b)
Fig. 1. (a) An axial magnetic coupling and (b) a radial magnetic coupling.

Manuscript received Nov. 20, 2016. Date of publication June, 2017. Corresponding authors: K. Li (email: kli10@uncc.edu), J. Z. Bird (e-mail: jonathan.bird@ieee.org).

Digital Object Identifier (inserted by IEEE).

\section{RAdial PERMAnent MAgnet COUPLING}

Consider the case in which a fictitious magnetic charge distribution described by a charge density function, $\rho_{m}\left(\theta_{I}\right)$, is distributed over the surface of a cylinder, such as sketched in Fig. 3(a). In this case the magnetic scalar potential field emanating from a charge cylinder, with radii $r_{o}^{I}$, can be computed from [9]

$$
\phi^{I}(r, \theta, z)=\frac{1}{4 \pi \mu_{0}} \int_{0}^{2 \pi} \int_{-d_{r} / 2}^{d_{r} / 2} \frac{\rho_{m}^{I}\left(\theta_{I}\right)}{R} r_{o}^{I} d z_{I} d \theta_{I}
$$

where $\mu_{o}=$ permittivity of free space, $d_{r}=$ axial rotor width and

$$
\begin{aligned}
R\left(r, \theta, \theta_{I}, z_{I}\right) & =\sqrt{C\left(r, r_{o}^{I}, \theta-\theta_{I}\right)+\left(z-z_{I}\right)^{2}} \\
C(a, b, \varphi) & =a^{2}+b^{2}-2 a b \cos (\varphi)
\end{aligned}
$$

It can be noted that at the axial center $(z=0)$ of the radial Halbach rotor shown in Fig. 2(b), the $B_{z}$ field is zero such that

$$
\mathbf{B}(r, \theta, 0)=B_{r}(r, \theta, 0) \hat{\mathbf{r}}+B_{\theta}(r, \theta, 0) \hat{\boldsymbol{\theta}}+0 \widehat{\mathbf{z}}
$$

In [10] it was shown that when (4) is satisfied the 3-D external field (where $r>r_{o}^{I}$ ) of a Halbach rotor can be accurately modeled by setting the charge density function in (1) to

$$
\rho_{m}(\theta)=2 B_{m r}^{I}\left(r_{o}^{I}\right) \cos (p \theta)
$$

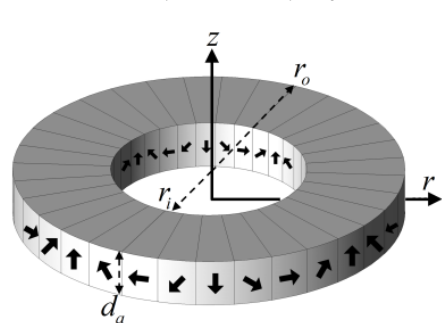

(a)

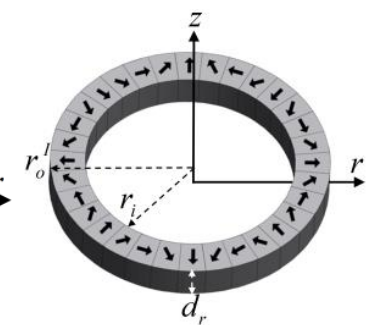

(b)
Fig. 2. (a) An 8-segment 4 pole-pair $z$-axis directed $\left(z>d_{a} / 2\right)$ axial Halbach rotor and (b) and externally directed $\left(r>r_{o}{ }^{I}\right)$ radial Halbach rotor

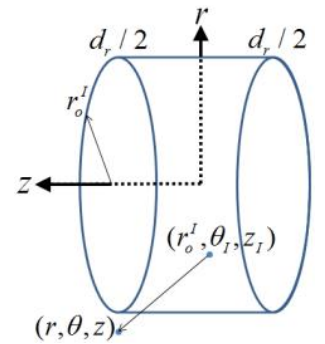

(a)

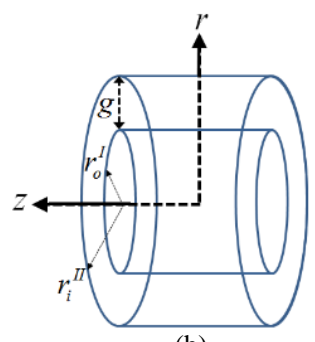

(b)
Fig. 3.(a) 3-D charge sheet model, $\left(r_{o}{ }^{I}, \theta_{I}, z_{I}\right)$ is a point located on the outer cylinder surface and (b) magnetic charge sheet coupling model where the two Halbach rotors are replaced with two fictious charge sheet cylinders. 
where [11]

$$
B_{m r}^{I}(r)=\frac{2 B_{m} p\left(1+\mu_{r}\right)\left[r_{i}^{p+1}-\left(r_{o}^{I}\right)^{p+1}\right]\left(r_{o}^{I}\right)^{2 p}}{r^{p+1}(1+p)\left[\left(1-\mu_{r}\right)^{2} r_{i}^{2 p}-\left(1+\mu_{r}\right)^{2}\left(r_{o}^{I}\right)^{2 p}\right]}
$$

and $\mu_{r}=$ magnet relative permittivity, $B_{m}=$ magnet residual flux density, $p=$ pole-pairs and $r_{i}=$ inner radii, as shown in Fig 2(b).

By substituting (5) into (1) and evaluating the $z$-axis integral the magnetic scalar potential can be determined to be [12]

$$
\begin{aligned}
& \phi^{I}(r, \theta, z)=\frac{B_{m r}^{I}\left(r_{o}^{I}\right) r_{o}^{I}}{2 \pi \mu_{0}} \int_{0}^{2 \pi} \cos \left(p_{I} \theta_{I}\right) \\
& \quad \times \log \left[\frac{2 z+d_{r}+\sqrt{\left(2 z+d_{r}\right)^{2}+4 C\left(r, r_{o}^{I}, \theta-\theta_{I}\right)}}{2 z-d_{r}+\sqrt{\left(2 z-d_{r}\right)^{2}+4 C\left(r, r_{o}^{I}, \theta-\theta_{I}\right)}}\right] d \theta_{I}
\end{aligned}
$$

Defining

$$
\theta_{d}=\theta-\theta_{I}
$$

one can then substitute (8) into (7) to obtain

$$
\begin{aligned}
& \phi^{I}(r, \theta, z)=-\frac{B_{m r}^{I}\left(r_{o}^{I}\right) r_{o}^{I}}{2 \pi \mu_{0}} \int_{0}^{2 \pi} \cos \left(p_{I} \theta-p_{I} \theta_{d}\right) \\
& \quad \times \log \left[\frac{2 z+d_{r}+\sqrt{\left(2 z+d_{r}\right)^{2}+4 C\left(r, r_{o}^{I}, \theta_{d}\right)}}{2 z-d_{r}+\sqrt{\left(2 z-d_{r}\right)^{2}+4 C\left(r, r_{o}^{I}, \theta_{d}\right)}}\right] d \theta_{d}
\end{aligned}
$$

The radial Halbach PMC can be replaced with two equivalent magnetic charge sheets as illustrated in Fig. 3(b). The charge sheets are located at $r=r_{o}^{I}$ and $r=r_{i}^{I I}$. The external field created by the inner charge sheet is given by (9). The field created by the outer charge sheet must be directed inwards (for $r<$ $\left.r_{i}^{I I}\right)$. Therefore, by using the 2-D solution to an inward directed ideal Halbach rotor field [11]

$$
B_{m r}^{I I}(r)=\frac{2 B_{m} p\left(1+\mu_{r}\right)\left[r_{o}^{p-1}-\left(r_{i}^{I I}\right)^{p-1}\right]\left(r_{o}\right)^{p+1}}{(1-p)\left[\left(1-\mu_{r}\right)^{2}\left(r_{i}^{I I}\right)^{2 p}-\left(1+\mu_{r}\right)^{2} r_{o}^{2 p}\right]}\left(\frac{r}{r_{i}^{I I}}\right)^{p-1}
$$

the charge function on the outer charge sheet $I I$ will be [10]

$$
\rho_{m}^{I I}\left(\theta_{I I}\right)=2 B_{m r}^{I I}\left(r_{i}^{I I}\right) \cos \left[p\left(\theta_{I I}-\theta_{t}\right)\right]
$$

where $\theta_{t}=$ angular offset position of charge sheet field and $\theta_{I I}=$ angular position on outer charge cylinder surface.

\section{A. Radial Halbach Coupling Torque}

The energy contained on the outer charge cylinder is [13]

$$
W=\int_{0}^{2 \pi} \int_{-d_{r} / 2}^{d_{r} / 2} \phi^{I}\left(r_{i}^{I I}, \theta_{I I}, z\right) \rho_{m}^{I I}\left(\theta_{I I}\right) r_{i}^{I I} d z d \theta_{I I}
$$

the torque can then be computed from [13]

$$
T=\left.\frac{\partial W}{\partial \theta_{I I}}\right|_{\phi^{l}=\text { constant }}
$$

Substituting (12) into (13) enables the torque created on the surface of the outer charge cylinder to be evaluated [13]

$$
T_{r}\left(\theta_{t}\right)=\int_{0}^{2 \pi} \int_{-d_{r} / 2}^{d_{r} / 2} \phi^{I}\left(r_{i}^{I I}, \theta_{I I}, z\right) \frac{\partial \rho_{m}^{I I}\left(\theta_{I I}\right)}{\partial \theta_{I I}} r_{i}^{I I} d z d \theta_{I I}
$$

then substituting (9) and (11) into (14) one obtains after rearranging and simplifying

$$
T_{r}\left(\theta_{t}\right)=\frac{B_{m r}^{I}\left(r_{o}^{I}\right) B_{m r}^{I I}\left(r_{i}^{I I}\right) r_{o}^{I} r_{i}^{I I} p}{\mu_{0}} \sin \left(p \theta_{t}\right) \int_{0}^{2 \pi} \int_{-d_{r} / 2}^{d_{r} 2} \cos \left(p \theta_{I I}\right)
$$

$$
\times \log \left[\frac{2 z+d_{r}+\sqrt{4 C\left(r_{i}^{I I}, r_{o}^{I}, \theta_{I I}\right)+\left(2 z+d_{r}\right)^{2}}}{2 z-d_{r}+\sqrt{4 C\left(r_{i}^{I I}, r_{o}^{I}, \theta_{I I}\right)+\left(2 z-d_{r}\right)^{2}}}\right] d z d \theta_{I I}
$$

Finally, evaluating the $z$-axis integral term in (15) yields

$$
\begin{aligned}
T_{r}\left(\theta_{t}\right)= & \frac{2 B_{m r}^{I}\left(r_{o}^{I}\right) B_{m r}^{I I}\left(r_{i}^{I I}\right) r_{o}^{I} r_{i}^{I I} p}{\mu_{0}} \sin \left(p \theta_{t}\right) \int_{0}^{2 \pi} \cos \left(p \theta_{I I}\right) \times \\
& \left(\sqrt{C\left(r_{i}^{I I}, r_{o}^{I}, \theta_{I I}\right)}-\sqrt{C\left(r_{i}^{I I}, r_{o}^{I}, \theta_{I I}\right)+d_{r}^{2}}\right. \\
+ & \left.\frac{d_{r}}{2} \log \left[\frac{d_{r}+\sqrt{C\left(r_{i}^{I I}, r_{o}^{I}, \theta_{I I}\right)+d_{r}^{2}}}{-d_{r}+\sqrt{C\left(r_{i}^{I I}, r_{o}^{I}, \theta_{I I}\right)+d_{r}^{2}}}\right]\right) d \theta_{I I}
\end{aligned}
$$

If (16) is compared to the torque coupling equations derived in [2-5] it can be noted that (16) is comparatively simple in form. Note that the air-gap length, $g$, is present within (16)

$$
r_{i}^{I I}=r_{o}^{I}+g
$$

\section{B. Model Validation}

Using the values shown in Table I the torque computed by (16) was compared with a COMSOL FEA model and a JMAG FEA model. The torque comparison is shown in Fig. 4. It can be noted that a close agreement was obtained.

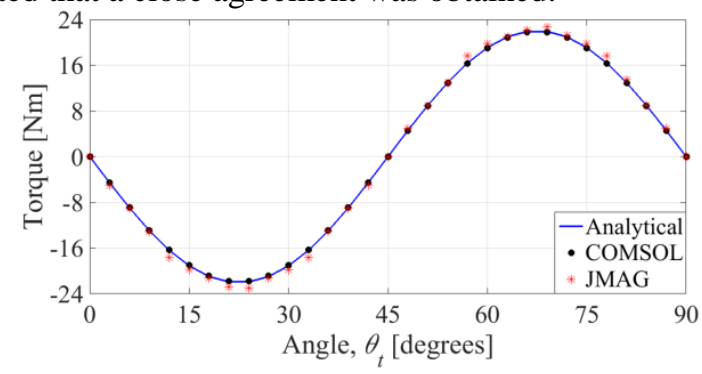

Fig. 4. Torque comparison between the analytical model and FEA models.

\section{IDEAL AXIAL HALBACH ROTOR COUPLING}

The torque created by an ideal axial Halbach PMC rotor was derived in [8] from the fundamental field component and can be written as a single double integral equation:

$$
\begin{aligned}
T_{a}\left(\theta_{t}\right) & =\sin \left(p \theta_{t}\right) \frac{B_{A m}^{2}}{\mu_{0}} p\left(\int_{0}^{2 \pi} \int_{r_{i}}^{r_{0}} \cos \left(p \theta_{d}\right) R_{d}\left(r, \theta_{d}\right) r d r d \theta_{d}\right. \\
& \left.+\int_{0}^{2 \pi} \int_{r_{i}}^{r_{a}} \cos \left(p \theta_{d}\right) \cos \left(\theta_{d}\right) \log \left[R_{e}\left(r, \theta_{d}\right)\right] r^{2} d r d \theta_{d}\right)
\end{aligned}
$$

where

$$
\begin{gathered}
R_{d}\left(r, \theta_{d}\right)=\sqrt{C\left(r, r_{o}, \theta_{d}\right)+g^{2}}-\sqrt{C\left(r, r_{i}, \theta_{d}\right)+g^{2}} \\
R_{e}\left(r, \theta_{d}\right)=\frac{r_{o}-r \cos \left(\theta_{d}\right)+\sqrt{C\left(r, r_{o}, \theta_{d}\right)+g^{2}}}{r_{i}-r \cos \left(\theta_{d}\right)+\sqrt{C\left(r, r_{i}, \theta_{d}\right)+g^{2}}} \\
B_{A m}=2 B_{r} \frac{\left(\mu_{r}+1\right) e^{k}\left(e^{k}-e^{-k}\right)}{e^{2 k}\left(\mu_{r}+1\right)^{2}-e^{-2 k}\left(\mu_{r}-1\right)^{2}} \\
k=p d_{a} /\left(r_{o}+r_{i}\right)
\end{gathered}
$$

The axial length, inner radii and outer radii expressed in (19)-(22) are defined as shown in Fig. 1(a). 
TABLE I

RADIAL COUPLING MATERIAL AND GEOMETRIC PARAMETERS

\begin{tabular}{|c|l|c|c|}
\hline Description & Value & Unit \\
\hline \multirow{3}{*}{$\begin{array}{c}\text { Rotor I } \\
\text { - inner } \\
\text { rotor }\end{array}$} & Outer radius, $r_{o}^{I}$ & 40 & $\mathrm{~mm}$ \\
\cline { 2 - 4 } & Inner radius, $r_{i}$ & 30 & $\mathrm{~mm}$ \\
\cline { 2 - 4 } & Axial length, $d_{r}$ & 10 & $\mathrm{~mm}$ \\
\cline { 2 - 4 } & Pole-pairs, $p$ & 4 & - \\
\hline \multirow{3}{*}{$\begin{array}{c}\text { Rotor II } \\
\text { - outer } \\
\text { rotor }\end{array}$} & Outer radius, $r_{o}$ & 51 & $\mathrm{~mm}$ \\
\cline { 2 - 4 } & Inner radius, $r_{i}^{I I}$ & 41 & $\mathrm{~mm}$ \\
\cline { 2 - 4 } & Axial length, $d_{r}$ & 10 & $\mathrm{~mm}$ \\
\cline { 2 - 4 } & Pole pairs, $p$ & 4 & - \\
\hline Magnetic permeability, $\mu_{r}$ & 1.05 & - \\
\hline \multicolumn{2}{|c|}{ Remnant flux density, $B_{m r}$} & 1.27 & $\mathrm{~T}$ \\
\hline \multicolumn{2}{|c|}{ Magnet material density, $\rho$} & 7,600 & $\mathrm{~kg} / \mathrm{m}$ \\
\hline \multicolumn{2}{|c|}{ Air gap, $g$} & 1 & $\mathrm{~mm}$ \\
\hline
\end{tabular}

IV. TORQue DENSITY

To better understand the fundamental geometric sizing relationship for the ideal axial and radial PMCs the mass and volumetric torque density equations are used. The mass torque densities for both the radial and axial PMCs are given by:

$$
\begin{aligned}
T_{R m}= & T_{r} /\left[\rho \pi d_{r}\left(r_{o}^{2}-\left(r_{i}^{I I}\right)^{2}+\left(r_{o}^{I}\right)^{2}-r_{i}^{2}\right)\right],[\mathrm{Nm} / \mathrm{kg}] \\
& T_{A m}=T_{a} /\left[\rho \pi\left(r_{o}^{2}-r_{i}^{2}\right) 2 d_{a}\right],[\mathrm{Nm} / \mathrm{kg}]
\end{aligned}
$$

where $\rho=$ density of magnet material. The volumetric torque density for the radial and axial PMC is given respectively by

$$
\begin{gathered}
T_{R v}=T_{r} /\left(d_{r} \pi r_{o}^{2}\right),\left[\mathrm{Nm} / \mathrm{m}^{3}\right] \\
T_{A v}=T_{a} /\left[\left(2 d_{a}+g\right) \pi r_{o}^{2}\right],\left[\mathrm{Nm} / \mathrm{m}^{3}\right] .
\end{gathered}
$$

\section{RADIAL COUPLING ANALYSIS}

The PMC inner radii and outer radii ratio is defined as

$$
\Lambda=r_{i} / r_{o}
$$

and the air-gap radii between rotors will be defined as

$$
r_{g}=r_{o}^{I}+g / 2
$$

With definition (28) the torque density for the radial PMC will be dependent on six parameters, namely, outer PMC radii, $r_{o}$, inner PMC radii, $r_{i}$, air-gap radii, $r_{g}$, pole-pairs, $p$, axial length, $d_{r}$, and air-gap $g$.

The influence of air-gap length on torque is self-evident and will be kept at $g=1 \mathrm{~mm}$. This is a very small air-gap for a practical PMC. However, the primary purpose of this paper is to determine the upper torque density bound for a magnetic device and therefore a $1 \mathrm{~mm}$ air-gap is not small for many other types of rotary magnetic devices.

The increase in the axial length $d_{r}$ will result in an increasing torque density, this effect is illustrated in Fig. 5. Fig. 5 also shows that for a given $r_{o}$ a further increase in $d_{a}$ will have a diminishing return. For instance, when $d_{r}>r_{o}$ the mass torque density will increase further by only $1 \%$ per-mm. Based on this analysis the axial length of the PMC will be scaled with PMC outer radii by keeping $d_{r}=r_{o}$.

If the $p$ and $r_{o}$ are now set to $\left(p, r_{o}\right)=(4,30 \mathrm{~mm})$ a torque density plot when $r_{i}$ and $r_{g}$ are varied can be created. The resultant plot is shown in Fig. 6. From this plot it can be noted that the peak volumetric and mass torque density occurs when $\left(r_{i}, r_{g}\right)=(0,21.5) \mathrm{mm}$ and $\left(r_{i}, r_{g}\right)=(15,23.5) \mathrm{mm}$ respectively. These locations are marked in Fig. 6 by a black dot.

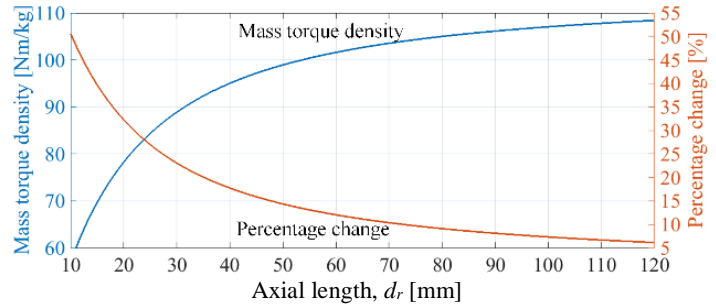

Fig. 5. The increase in mass torque density when the axial length $d_{r}$ is increased while holding the other values in Table I constant.

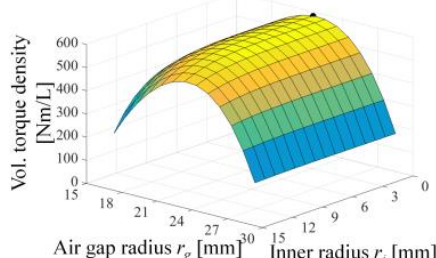

(a)

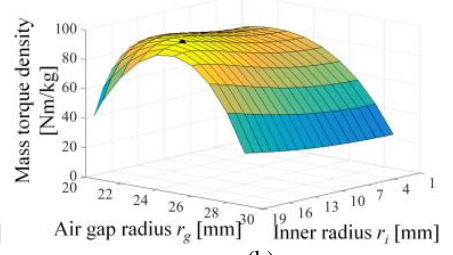

(b)
Fig. 6.(a) Volumetric and (b) mass torque density when $r_{g}$ and $r_{i}$ are both varied and $p=4, g=1 \mathrm{~mm}, r_{o}=30 \mathrm{~mm}, d=r_{o}$.

The value of $\left(r_{i}, r_{g}\right)$ that gives the peak mass torque density for each different $r_{o}$ and $p$ value was then calculated, the resulting peak mass and volumetric torque density plots are shown in Fig. 7(a) and (b) while Fig. 7(c) show the corresponding $r_{g}$ used to achieve the peak values.

By studying Fig. 7(c) it was determined that the peak mass torque density always occurs when

$$
r_{g}=\left(r_{i}+r_{o}\right) / 2
$$

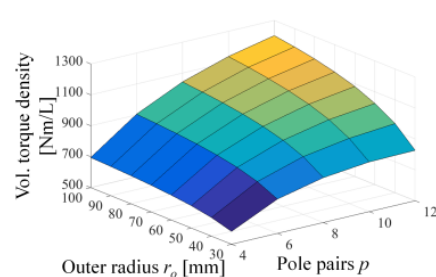

(a)

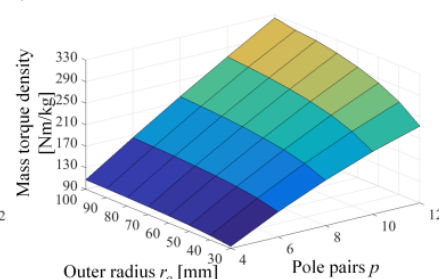

(b)

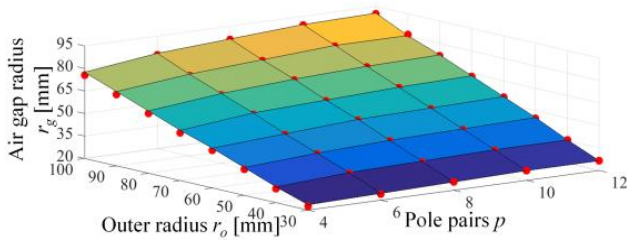

(c)

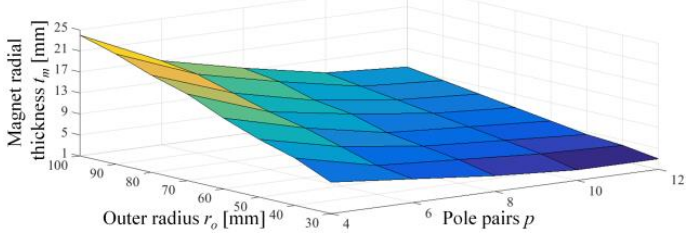

(d)

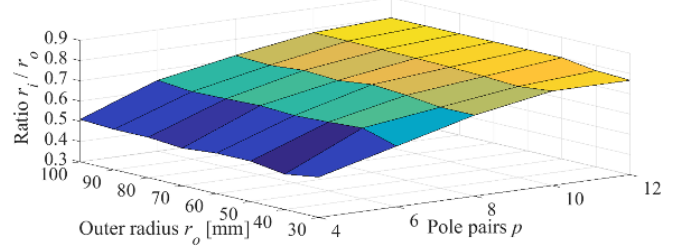

(e)

Fig. 7. (a) Volumetric and (b) mass torque density for different pole-pairs and outer radii also shown is (c) corresponding $r_{g}$ value, (d) corresponding magnet radial thickness, $t_{m}$ and (e) inner-to-outer PMC ratio, $\Lambda$ for the peak mass torque density condition. 
with (29) satisfied the magnet radial thickness must then be

$$
t_{m}=\left(r_{o}-r_{i}-g\right) / 2
$$

The corresponding magnet thickness value at each peak torque condition is shown in Fig. 7(d). It can be noted from Fig. 7(d) that as $p$ increases the optimal magnet thickness, $t_{m}$ decreases and this leads to a higher torque density, while Fig. 7(e) shows that the $\Lambda$-ratio is a constant with the same value of polepairs, $p$. The optimal $\Lambda$ calculated for different numbers of polepairs is shown in Fig. 8 . The $\Lambda$-ratio can be curved fitted and is related to the number of pole-pairs by

$$
\Lambda=1-10 /\left(3+5 p^{0.9}\right)
$$

By examining Fig. 7, and noting that the magnetic shear stress is independent of $p$, it was determined that at peak mass torque density the following condition is always satisfied

$$
r_{o}-t_{m}=p t_{m} \pi / 4
$$

The condition given by (32) can be confirmed by considering the plot shown in Fig 9. in which $r_{o}$ and $t_{m}$ were both varied whilst hold $p=4$. The line at which (32) is satisfied is also shown in Fig. 9, the line clearly traverses the peak.

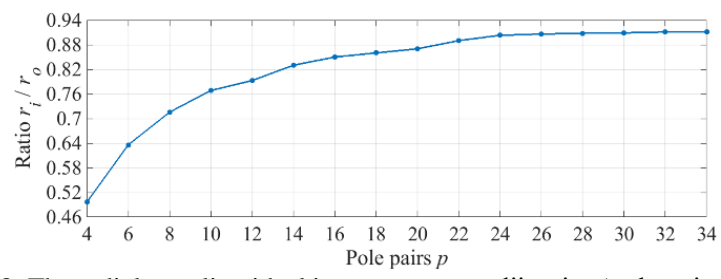

Fig. 8. The radial coupling ideal inner-to-outer radii ratio, $\Lambda$, that gives the peak mass torque density for different numbers of pole-pairs.

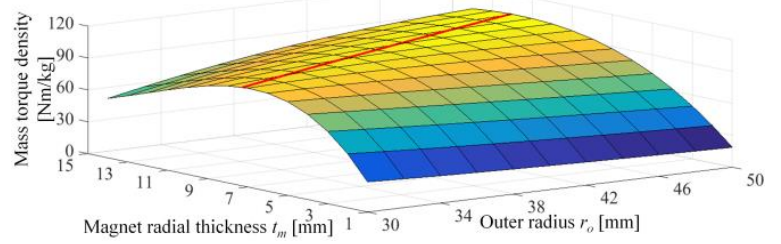

Fig. 9. Variation of mass torque density when $r_{o}$ and $t_{m}$ are varied and $p=4$, $d_{r}=r_{o}, g=1 \mathrm{~mm}$. The line that satisfies (32) is superimposed on the plot.

\section{AXIAL AND RADIAL COUPLING COMPARISON}

Keeping $r_{o}$ and $r_{i}$ fixed at the optimal value calculated for the radial PMC the axial PMC mass and volumetric torque density was then computed by using (18). The $d_{a}$ and $p$ values were selected to achieve the peak mass torque density [14]. The resultant plot is shown in Fig. 10.

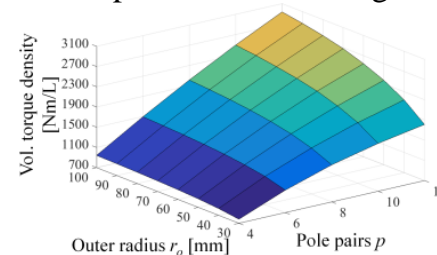

(a)

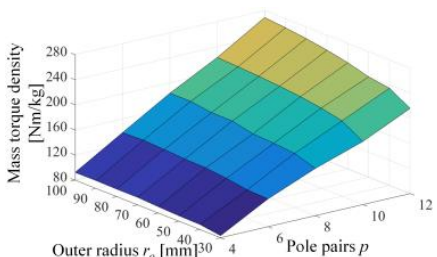

(b)
Fig. 10. (a) Volumetric and (b) mass torque density for the axial PMC for different pole-pairs and outer radii when $r_{i}$ is the same as used by the radial PMC and $g=1 \mathrm{~mm}$. The axial length $d_{a}$ was selected so as to meet the peak mass torque density condition for an ideal axial PMC [14].

Comparing Fig. 10 with Fig. 7(a)-(b) it can be noted that the radial PMC can operate at a higher volumetric torque density whilst the AMC can achieve a higher mass torque density.

\section{CONCLUSION}

An ideal radial PMC torque equation was derived for the condition in which the source field was assumed to be created by an ideal Halbach rotor (a single fundamental field component). The derived torque equation is relatively easy to evaluate as only a single integral needs to be evaluated. The fundamental interrelationship between outer radii, magnet thickness and pole-pairs was determined that yields the peak mass torque density condition. A comparative analysis with an ideal axial PMC showed that the radial PMC had a higher volumetric torque density while the axial PMC attained a higher mass torque density.

\section{ACKNOWLEDGMENTS}

The authors would gratefully like to thank the JMAG corporations for the use of their FEA software. This material is based upon work supported by the Department of Energy under Grant No. DE-EE0006801 as well as a North Carolina Ocean Energy Grant.

\section{REFERENCES}

[1] P. O. Rasmussen, T. O. Andersen, et al., "Development of a highperformance magnetic gear," IEEE Trans. Ind. Appl., vol. 41, pp. 764$770,2005$.

[2] R. Ravaud and G. Lemarquand, "Magnetic couplings with cylindrical and plane air gaps: influence of the magnet polarization direction," Prog. Electromagnetics Res. B, vol. 16, pp. 333-349, 2009.

[3] T. R. N. Mhiochain, D. Weaire, et al., "Analysis of torque in nested magnetic cylinders," J. Appl. Phy., vol. 86, pp. 6412-6424, 1999.

[4] H.-B. Kang, J.-Y. Choi, et al., "Comparative study of torque analysis for synchronous permanent magnet coupling with parallel and Halbach magnetized magnets based on analytical field calculations," IEEE Trans Magn., vol. 50, p. Article \# 8600604, 2014.

[5] J.-F. Charpentier and G. Lemarquand, "Optimal design of cylindrical airgap synchronous permanent magnet couplings," IEEE Trans. Mag., vol. 35, pp. 1037-1046, Mar. 1999.

[6] W. Y. Lin, L. P. Kuan, et al., "Near-optimal design and 3-D finite element analysis of multiple sets of radial magnetic couplings," IEEE Trans. Magn., vol. 44, pp. 4747-4753, Dec. 2008.

[7] S. Högberg, B. B. Jensen, et al., "Improving torque per kilogram magnet of permanent magnet couplings using finite element analysis," in Inter. Elec. Mach. Drives Conf. (IEMDC), Chicago, IL, May, 2013, pp. pp. 1074-1079.

[8] K. Li and J. Z. Bird, "A 3-D analytical model of a Halbach axial magnetic coupling," in Inter. Sym. Power Elect. Elect. Drives Auto. Motion (SPEEDAM), Anacapri, Italy, 2016, pp. 1448-1454.

[9] H. L. Rakotoarison, J.-P. Yonnet, et al., "Using coulombian approach for modeling scalar potential and magnetic field of a permanent magnet with radial polarization," IEEE Trans. on Mag., vol. 43, pp. 1261-1264, 2007.

[10] S. Paul, D. Bobba, et al., "Source field modeling in air using magnetic charge sheets," IEEE Trans. Mag., vol. 48, pp. 3879-3882, 2012.

[11] Z. P. Xia, Z. Q. Zhu, et al., "Analytical magnetic field analysis of halbach magnetized permanent-magnet machines," IEEE Trans. Magn., vol. 40, pp. 1864-1872, July 2004.

[12] V. M. Acharya, "Design of a flux focusing axial magnetic gear," M.S. Thesis, Elect. and Comp. Eng., Univ. North Carolina at Charlotte, Charlotte, USA, Dec. 2013.

[13] O. D. Jefimenko, Electricity and Magnetism. New York: Meredith Publishing Co., 1966.

[14] K. Li and J. Z. Bird, "Scaling equations for an axial magnetic coupling," Submitted to IEEE Trans Magn., 2016. 